\title{
A complex resistance locus in Solanum americanum recognizes a conserved Phytophthora effector
}

Kamil Witek ${ }^{1 \#}$, Xiao Lin ${ }^{1 \#}$, Hari S Karki ${ }^{1 \# \$}$, Florian Jupe ${ }^{1 \$}$, Agnieszka I Witek ${ }^{1}$, Burkhard Steuernagel $^{2}$, Remco Stam ${ }^{3}$, Cock van Oosterhout ${ }^{4}$, Sebastian Fairhead ${ }^{1}$, Robert Heal $^{1}$, Jonathan M Cocker ${ }^{5,6}$, Shivani Bhanvadia ${ }^{7}$, William Barrett ${ }^{1 \$}$, ChihHang $\mathrm{Wu}^{1 \$}$, Hiroaki Adachi ${ }^{1}$, Tianqiao Song ${ }^{1 \$}$, Sophien Kamoun ${ }^{1}$, Vivianne GAA Vleeshouwers ${ }^{7}$, Laurence Tomlinson ${ }^{1}$, Brande BH Wulff ${ }^{2}$ and Jonathan DG Jones ${ }^{1 *}$

${ }^{1}$ The Sainsbury Laboratory, University of East Anglia, Norwich Research Park, Norwich, NR4 7UH, UK

${ }^{2}$ John Innes Centre, Norwich Research Park, Norwich, NR4 7UH, UK

${ }^{3}$ Phytopathology, Technical University Munich, 85354 Freising, Germany

${ }^{4}$ School of Environmental Sciences, University of East Anglia, Norwich Research Park, Norwich, NR4 7TJ, UK

${ }^{5}$ Faculty of Biological Sciences, University of Leeds, Leeds, LS2 9JT, UK

${ }^{6}$ University of Hull, Hull, HU6 7RX, UK

${ }^{7}$ Plant Breeding, Wageningen University and Research, Droevendaalsesteeg 1, 6708 PB, Wageningen, The Netherlands

${ }^{\#}$ These authors contributed equally to this work

${ }^{\$}$ Current addresses:

HSK: U.S. Department of Agriculture-Agricultural Research Service, Madison, WI 53706, U.S.A

FJ: Bayer Crop Science, Chesterfield, MO, USA

WB: The New Zealand Institute for Plant \& Food Research Ltd, Nelson, New Zealand

CHW: Institute of Plant and Microbial Biology, Academia Sinica, Taiwan

TS: Institute of Plant Protection, Jiangsu Academy of Agricultural Sciences, Nanjing, 210014, P. R. China

*Corresponding author: Jonathan D. G. Jones (jonathan.jones@tsl.ac.uk) 


\begin{abstract}
Late blight caused by Phytophthora infestans greatly constrains potato production. Many Resistance $(R)$ genes were cloned from wild Solanum species and/or introduced into potato cultivars by breeding. However, individual $R$ genes have been overcome by $P$. infestans evolution; durable resistance remains elusive. We positionally cloned a new $R$ gene, Rpi-amrl, from Solanum americanum, that encodes an NRC helperdependent CC-NLR protein. Rpi-amrl confers resistance in potato to all 19 P. infestans isolates tested. Using association genomics and long-read RenSeq, we defined eight additional Rpi-amrl alleles from different $S$. americanum and related species. Despite only $\sim 90 \%$ identity between Rpi-amr1 proteins, all confer late blight resistance but differentially recognize Avramrl orthologs and paralogs. We propose that Rpi-amrl gene family diversity assists detection of diverse paralogs and alleles of the recognized effector, facilitating durable resistance against $P$. infestans.
\end{abstract}




\section{Introduction}

Potato is the third most important directly-consumed food crop world-wide ${ }^{1}$. Phytophthora infestans, an oomycete pathogen, causes late blight disease in potato, and can result in complete crop failure. Disease management is primarily based on repeated fungicide applications (10-25 times per season in Europe). However, fungicideresistant races have emerged ${ }^{2}$.

To elevate late blight resistance, Resistance to Phytophthora infestans (Rpi) genes were identified in wild relatives of potato and used for resistance breeding ${ }^{3}$. More than 20 $R p i$ genes have been mapped and cloned from different Solanum species, e.g. R2 (Rpiblb3), R3a, R8, Rpi-blb1, Rpi-blb2 and Rpi-vnt1 ${ }^{4-10}$. All encode coiled-coil (CC), nucleotide binding (NB), leucine-rich repeat (LRR) (NLR) proteins ${ }^{11}$ and some require helper NLR proteins of the NRC family ${ }^{12}$. However, most cloned Rpi genes can be overcome by at least one strain of $P$. infestans ${ }^{13}$. Provision of durable late blight resistance for potato remains a major challenge.

NLR-mediated immunity upon effector recognition activates "effector-triggered immunity" $(\mathrm{ETI})^{14}$. In oomycetes, all identified recognized effectors, or avirulence (AVR) proteins, carry a signal peptide and an RXLR motif ${ }^{15} .563$ RxLR effectors were predicted from the $P$. infestans genome, enabling identification of the recognized effectors ${ }^{16,17}$. Many $P$. infestans effectors show signatures of selection to evade recognition by corresponding NLR proteins ${ }^{18}$. NLR genes also show extensive allelic and presence/absence variation in wild plant populations ${ }^{19,20}$ and known Resistance $(R)$ gene loci like Mla, L, Pi9, RPPI and RPP13 from barley, flax, rice and Arabidopsis show substantial allelic polymorphism ${ }^{21-24}$. Remarkably, different barley Mla and flax $L$ gene alleles can recognize sequence-unrelated effectors ${ }^{25,26}$.

Technical advances like RenSeq (Resistance gene enrichment and Sequencing) and PenSeq (Pathogen enrichment Sequencing) enable rapid definition of allelic variation and mapping of plant NLRs, or discovery of variation in pathogen effectors ${ }^{27-29}$. Combined with single-molecule real-time (SMRT) sequencing, SMRT RenSeq enabled cloning of Rpi-amr3 from Solanum americanum ${ }^{30}$. Similarly, long read and cDNA 
PenSeq enabled us to identify Avramrl from P. infestans ${ }^{31}$.

In this study, we further explored the genetic diversity of $S$. americanum, and by applying sequence capture technologies, we fine-mapped and cloned Rpi-amrl from $S$. americanum, (usually) located on the short arm of chromosome 11. Multiple Rpi-amrl homologs were found in different $S$. americanum accessions and in relatives, including Solanum nigrescens and Solanum nigrum. Functional alleles show extensive allelic variation and confer strong resistance to all 19 tested diverse P. infestans isolates. Although differential recognition was found between different Rpi-amrl and Avramrl homologs, all Rpi-amrl alleles recognize the Avramrl homologs from Phytophthora parasitica and Phytophthora cactorum. Our study reveals unique properties of genetic variation of $R$ genes from "non-host" species.

\section{Results}

\section{Rpi-amr1 maps to the short arm of chromosome 11}

We previously investigated $S$. americanum and isolated Rpi-amr3 from an accession 944750095 (SP1102) $^{30}$. To discover new Rpi-amr genes, we characterized additional 14 lines of $P$. infestans-resistant $S$. americanum and close relatives $S$. nigrescens and Solanum nodiflorum by crossing them to a susceptible (S) S. americanum line 954750186 (hereafter SP2271) (Table 1, Fig. S1). To avoid self-pollination, a resistant parent was always used as a pollen donor. All the corresponding F1 plants (6-10 per cross) were resistant in a detached leaf assay (DLA) (Table 1). Around 60-100 F2 progeny derived from each self-pollinated F1 plant were phenotyped by DLA using $P$. infestans isolate $88069^{32}$. The $\mathrm{F} 2$ progenies that derived from the resistant parents with working numbers SP1032, SP1034, SP1123, SP2272, SP2273, SP2360, SP3399, SP3400, SP3406, SP3408 and SP3409 segregated in a ratio suggesting the presence of a single (semi-) dominant resistance gene (fitting 3:1 or 2:1 [likely due to segregation distortion], R:S - resistant to susceptible - ratio). Two crosses showed a 15:1 segregation (resistant parent SP2300 and SP2307), suggesting the presence of two unlinked resistance genes. Investigating resistance in SP1101 required two backcrosses to SP2271 prior to selfing of resistant progeny to reveal a 3:1 R:S segregation. 
To identify Rpi genes from these resistant S. americanum accessions, we prioritized an F2 population derived from resistant parent SP2273 and named the corresponding gene Rpi-amrl. Using markers from RenSeq, genotyping by sequencing (RAD markers) and Whole Genome Shotgun sequencing (WGS), the Rpi-amrl gene was mapped in a small population ( $\mathrm{n}=188$ gametes) to the short arm of chromosome 11, between markers RAD_3 and WGS_1 (Fig. 1a, Table S1,S2). We expanded the mapping population and developed a PCR marker WGS_2 that co-segregated with resistance in 3,586 gametes (Fig. 1b, Table S2). To generate the physical map of the target interval from SP2273, a BAC library was generated. Two BAC clones $(12 \mathrm{H}$ and $5 \mathrm{G})$ covering the target interval were identified by a PCR screen with the above linked marker, sequenced on the PacBio RSII platform, and assembled into a single contig of 204,128 bp (Fig. 1c). We predicted 11 potential coding sequences on the assembled contig, nine of which encode $N L R$ genes (Fig. 1c). These NLR genes belong to the CNL class and have $80-96 \%$ betweenparalog identity.

To define which of these $N L R$ genes are expressed, cDNA RenSeq data of the resistant parent SP2273 were generated and mapped to the BAC_5G sequence. Seven out of nine $N L R$ genes were expressed. These genes - Rpi-amrla, b, $c, d, e, g$ and $h$ - were tested as candidate genes for Rpi-amrl (Fig. 1c).

\section{Rpi-amrle confers resistance in Nicotiana benthamiana and cultivated potato}

To test the function of the seven candidate genes, we cloned their open reading frames from genomic DNA inclusive of introns into a binary expression vector under control of the $35 \mathrm{~S}$ promoter. Rpi-amr3 was used as a positive control and the non-functional Rpi-amr3-S was used as a negative control. The constructs carrying each of the seven candidate genes were transiently expressed after Agrobacterium infiltration into $N$. benthamiana leaves, which were subsequently inoculated with the P. infestans isolate 88069 as described previously ${ }^{30}$. P. infestans growth was observed six days post inoculation (dpi). Only 35S::Rpi-amrle-infiltrated leaves showed reduced pathogen growth at 6 dpi compared to other candidate genes like Rpi-amrlc, or negative control Rpi-amr3-S. (Fig. 1e). Hence, we conclude that Rpi-amrle is the functional Rpi-amr1 (hereafter) gene from S. americanum SP2273. 
To test if Rpi-amrl confers late blight resistance in potato, we cloned it with its native promoter and terminator, and generated transgenic potato cultivar Maris Piper plants carrying Rpi-amr1. A non-functional paralog Rpi-amrla was also transformed into Maris Piper as a negative control. As in the transient assay, stably transformed Rpiamrl lines resisted $P$. infestans 88069 in potato (Fig. 1f), but Rpi-amrla-transformed plants did not (Fig. 1g).

\section{Rpi-amrl is a four exon CC-NLR}

To characterize the structure of Rpi-amrl, we mapped the cDNA RenSeq data to the full length Rpi-amrl gene and found four alternatively spliced forms of Rpi-amrl. The most abundant form, supported by $>80 \%$ of reads, comprises four exons encoding a protein of 1,013 amino acids. The remaining three forms had shifts in reading frames, leading to premature stop codons or absence of some exons. This was confirmed with 3' RACE PCR (Fig. 1d). The Rpi-amr1 is a typical CC-NB-LRR resistance protein, with a coiled-coil domain (CC; amino acids 2-146), nucleotide binding domain (NBARC; amino acids 179-457) and leucine-rich repeats (LRR; located between amino acids 504-900) which are all positioned in the first exon (1-918 aa, Fig. 2a). The remaining three short exons (amino acids 919-943, 944-1002 and 1,003-1,013) lack homology to any known domains. No integrated domains ${ }^{33}$ were found in the Rpi-amr1 protein.

\section{Functional Rpi-amr1 homologs are present in were identified from multiple resistant $S$. americanum and relatives}

Previously, we found at least 14 S. americanum accessions and related species that resist late blight (Table 1). To test if Rpi-amrl contributes to late blight resistance in other resistant $S$. americanum accessions, we genotyped 10-50 susceptible F2 plants of the populations derived from resistant accessions, with a marker positioned in Rpi-amrl gene (56766, Fig. 1 and Table S2). We found that the marker is absent in all tested susceptible descendants of accessions SP1032, SP1034, SP1123, SP2272, SP2307, SP2360, SP3399, SP3400, SP3406 and SP3408, suggesting that the resistance is linked to the Rpi-amrl locus. To test if in these accessions the resistance is conferred by functional Rpi-amrl homologs, we performed SMRT RenSeq-based de novo assembly 
of each resistant accession, and looked for homologs with the greatest identity to Rpiamrl. For accessions SP2307, SP3399 and SP3406, we also used cDNA RenSeq to monitor their expression. We mapped de novo contigs to the coding sequence of Rpiamrl allowing for $15 \%$ mismatches and gaps, and selected the closest homolog as a candidate Rpi-amrl ortholog (Table S3). In three resistant parents, namely SP1034, SP2360 and SP3400, the functional alleles showed 100\% identity at the amino acid level to Rpi-amr1, while amino acid sequences from the remaining accessions had as little as $89 \%$ identity to the functional Rpi-amr1 (Table S3). As described previously, we transiently expressed the closest related candidate Rpi-amrl homologs in $N$. benthamiana leaves followed by DLA with $P$. infestans isolate 88069 , and verified their functionality. The unique homologs of Rpi-amrl-2273 were named as Rpi-amr1-1032, Rpi-amr1-1123, Rpi-amr1-2272, Rpi-amr1-2307 and Rpi-amr1-3408.

For some accessions, like SP1101 and SP2300, the Rpi-amrl-linked markers gave ambiguous results, so we directly performed bulked segregant analysis (BSA) and RenSeq. Additional Rpi-amrl co-segregating paralogs, Rpi-amr1-1101 and Rpi-amrl2300, were identified and verified in transient assays as above (Fig. 2b).

Similarly, we inspected an F2 population derived from S. nigrescens accession SP3409 (Table 1). We applied BSA RenSeq and SMRT RenSeq to the resistant parents and F2 segregating population, and we found five candidate $N L R s$ belonging to the same $R p i$ amrl clade, all of which are expressed. The five candidates were cloned, and transient assays verified one of them as a functional Rpi-amrl homolog, Rpi-amrl-3409. However, Rpi-amr1-3409 does not co-segregate with Rpi-amrl-linked markers. We used GenSeq sequence capture-based genotyping (Chen et al. 2018), and found that Rpi-amr1-3409 locates on chromosome 1, based on the potato DM reference genome ${ }^{34}$. This result suggests that a fragment of DNA that locates on distal end of the short arm of chromosome 11 in other resistant accessions was translocated to the distal end of the long arm of chromosome 1 in SP3409.

When the full-length amino acid sequences of nine Rpi-amrl homologs were aligned, the polymorphisms between different functional alleles were found to be distributed through all domains including the LRR region (Fig. 2a and Fig. S2). 
Taken together, by using BSA RenSeq, SMRT RenSeq, cDNA RenSeq, association genomics and GenSeq, we cloned eight additional functional Rpi-amrl homologs from different resistant accessions, of which all confer resistance to P. infestans 88069 in transient assays. The closest Rpi-amrl homolog from susceptible parent SP2271 does not confer resistance (Fig. 2b).

\section{Rpi-amr1 confers broad-spectrum late blight resistance in cultivated potato}

To test the scope of late blight resistance conferred by Rpi-amrl and its homologs, we generated stably transformed transgenic potato cv Maris Piper plants carrying Rpiamr1-2272 and Rpi-amr1-2273, the most diverged of the homologs (Table S3), and inoculated them by DLA with 19 P. infestans isolates from UK, the Netherlands, Belgium, USA, Ecuador, Mexico and Korea (Table 2). Many of the tested P. infestans isolates can defeat multiple Rpi genes (Table 2). Our DLAs show that Maris Piper carrying Rpi-amr1-2272 or Rpi-amrl-2273 resist all 19 tested P. infestans isolates, while the wild-type Maris Piper control is susceptible to all of them. This indicates that Rpi-amrl confers broad-spectrum resistance against diverse $P$. infestans races.

\section{Differential recognition by Rpi-amr1 alleles of Avramr1 homologs}

Avramrl (PITG_07569) was identified in P. infestans race T30-4 by long-read and cDNA PenSeq, and multiple Avramrl homologs were identified in four P. infestans isolates and classified into four subclades ${ }^{31}$. To investigate if all nine cloned Rpi-amrl homologs could recognize diverse Avramrl homologs from different P. infestans isolates, in addition to Avramrl from race T30-4 that corresponds to clade A, we synthesized three Avramrl homologs Avramr1-13B1, Avramr1-13C2 and Avramr113D1 from isolate 3928A (EU_13_A2, commonly known as "Blue 13"), corresponding to clades B, C and D, respectively (Fig. 3). We also synthesized the Avramrl homologs from $P$. parasitica and $P$. cactorum $^{31}$. These six Avramrl homologs were co-expressed in $N$. benthamiana by agro-infiltration in all possible combinations with nine functional Rpi-amrl homologs and the non-functional Rpi-amrl-2271 as a negative control (Fig. $3)$. 
We found that different combinations of Rpi-amrl alleles and Avramrl homologs led either to strong, weak or no HR phenotype in transient assay, but the non-functional Rpi-amr1-2271 allele failed to recognize any Avramrl homologs (Fig. 3) The representative HR phenotype and the scoring of HR indices are shown in Fig. S3. Rpiamr1-2300 and Rpi-amrl-2307 recognized one Avramrl homolog each, but others detected Avramrl homologs from more than one clade. Clade C, represented here by Avramr1-13C2, is usually not expressed ${ }^{31}$, and when expressed from $35 \mathrm{~S}$ promoter, this effector was not recognized by most Rpi-amrl homologs, though a weak HR was observed upon co-expression with Rpi-amr1-2272. Avramr1-13D1 belongs to Clade D, which is absent in T30-4 but present in four other sequenced isolates ${ }^{31}$, and was recognized by all but one (Rpi-amr1-2300) homologs in the transient assay. Surprisingly, two Avramrl homologs from P. parasitica and P. cactorum are strongly recognized by all functional Rpi-amrl homologs, apart from Rpi-amrl-2272 which showed a weaker HR (Fig. 3).

Collectively, our data shows that Rpi-amrl/Avramrl homolog pairs provoke quantitatively and qualitatively different HRs, but all functional Rpi-amrl homologs detect at least one Avramrl homolog from P. infestans isolate 3928A.

\section{Both Rpi-amr1-mediated resistance and effector recognition are NRC2 or NRC3 dependent}

We generated a phylogenetic tree for representative Solanaceae NLR proteins. Rpiamr1 is grouped with clade CNL-3, from which no functional resistance genes were previously cloned (Fig. 4a). The closest related cloned functional gene is Rpi-amr3 (31.2\% identity on aa level) belonging to clade CNL-13 and located on chromosome 4. The phylogenetic affiliation suggested that Rpi-amr1 is likely to depend on the helper NRC clade because CNL-3 is among the large super-clade of NRC-dependent sensors (Fig. 4a) ${ }^{12}$.

To test this hypothesis, we transiently expressed Rpi-amr1-2273 together with PpAvramrl in NRC4, NRC2/3 or NRC2/3/4 knock out $N$. benthamiana leaves ${ }^{35,36}$ (Fig. S4). The HR phenotype was abolished in NRC2/3 and NRC2/3/4 knockout plants (Fig. S5c and b), but not in NRC4 knock-out or wild-type plants (Fig. S5d and a). The HR 
was recovered when NRC2 or NRC3 was co-expressed in the NRC2/3/4 or NRC2/3 knock out plants, but co-expression of NRC4 did not complement the loss of HR phenotype in NRC2/3/4 knockout plants. (Fig. S5b and c). We further showed that also Rpi-amrl mediated resistance is dependent on NRC2 or NRC3 but not NRC4, as transient expression of Rpi-amrl-2273 followed by P. infestans infection restricted pathogen growth only in NRC4 knockout $N$. benthamiana plants (Fig. 4b-d). These data indicate that both the effector recognition and resistance conferred by Rpi-amrl are NRC2 or NRC3 dependent.

\section{Functional Rpi-amr1 homologs are present in hexaploid $S$. nigrum accessions}

Most S. nigrum accessions are highly resistant to P. infestans and S. nigrum has been reported to be a "non-host" to $P$. infestans ${ }^{37}$, even though rare accessions are susceptible $^{38}$. S. americanum may be the diploid ancestor of hexaploid S. nigrum ${ }^{39}$. To test if Rpi-amrl also contributes to late blight resistance in S. nigrum, we designed nested PCR primers based on the Rpi-amrl-2273 sequence, and amplified and sequenced the coding sequence of Rpi-amrl homologs from two resistant and one reported susceptible $S$. nigrum accessions ${ }^{38}$. From two resistant accessions (SP1088 and SP1097; Table S4), we amplified sequences with $>99 \%$ nucleotide identity to $S$. americanum Rpi-amr1-2273, namely Rpi-nig1-1088 and Rpi-nig1-1097. The protein sequences of Rpi-nig1-1088 and Rpi-nig1-1097 are identical, with only one amino-acid (225 R->Q) change compared to Rpi-amr1-2273 (Fig. S6a). The primers used for allele mining did not amplify a product of the expected size for Rpi-amrl from the susceptible line SP999. To test their function, we performed transient assay for HR and disease resistance on $N$. benthamiana. We found both Rpi-nig1-1088 and Rpi-nig1-1097 show strong HR when co-expressed with PpAvramr1 and PcAvramr1. However, they activate a weaker HR to Avramr1 and Avramr1-13B1 compared to Rpi-amr1-2273 (Fig. S6b). Like Rpi-amr1-2273, but not the negative control Rpi-amr1-2271, transiently expressed Rpi-nig1-1088 and Rpi-nig1-1097 confer resistance to P. infestans 88069 (Fig. S6c). It is the first report of functional Rpi genes from S. nigrum, and our finding suggests the strong late blight resistance of S. nigrum is determined or partially determined by the Rpi-amrl homologs, that were most likely inherited from $S$. americanum. 


\section{High allelic diversity at Rpi-amr1 was generated through inter-paralog and ortholog sequence exchange}

Rpi-amrl alleles show relatively high nucleotide diversity ( $\pi=0.04)$, which could be an indication of balancing or diversifying selection (Table S5). In addition, Rpi-amrl alleles differ in their recognition of the Avramrl homologs (Fig. 3) which is also consistent with selection in a host-parasite co-evolutionary arms race. To test the hypothesis that allelic polymorphism at Rpi-amrl results from diversifying selection, we calculated diversity statistics and performed a McDonald-Kreitman test on both Rpiamrl alleles and Avramrl homologs. As expected, Avramrl homologs show a signature consistent with balancing selection (Tajima's D = 2.27) (Table S5). Remarkably, despite the high nucleotide diversity, no clear signals of balancing or diversifying selection were detected for Rpi-amrl (Tajima's D = 0.09083) (Table S5). Aligning the Rpi-amrl alleles against the reference and scrutinizing the sequences in more detail provided further insights. The nucleotide similarity of alleles varies markedly across the Rpi-amrl homologs (Fig. 2a and Table S3); this pattern is consistent with occasional recombination between highly diverged alleles or paralogs.

To test whether recombination could explain the observed polymorphisms in Rpi-amrl alleles, we predicted the possible recombination events using 3SEQ. Several recombination events were detected between Rpi-amrl orthologs from different $S$. americanum accessions, and Rpi-amrl paralogs from SP2273 (Table S6). Some sequence exchanges were visualized using HybridCheck (Fig. S7) ${ }^{40}$, and these data suggest that sequence exchange occurred between functional Rpi-amrl alleles and paralogs. To confirm these findings, we mapped all cloned Rpi-amrl CDS back to the BAC_5G sequence from accession SP2273 (Fig. S8). As expected, some Rpi-amrl homologs (e.g. SP2300 and SP2272) show a perfect match with the fourth NLR, and show a distribution of high identity that reflects the intron-exon structure. For some homologs (e.g. 2271), 5' end sequences match different NLR sequences on the BAC_5G and for others (e.g. 2275) part of the sequence is highly diverged from BAC_5G. Taken together, our results indicate that the polymorphism of Rpi-amrl alleles appears to have arisen partly due to sequence exchange between highly diverged alleles and paralogs, and not just through mutation accumulation. 


\section{Discussion}

Achieving complete and durable resistance is the ultimate goal of resistance breeding. Here, we report significant progress towards durable resistance against potato late blight. Most cloned late blight resistance genes derive from wild tuber-bearing species of genus Solanum, and many have been overcome by one or more P. infestans strains ${ }^{41}$. Conceivably, resistance to $P$. infestans in nearly all $S$. americanum and $S$. nigrum accessions is due to multiple $N L R$ genes, as zoospores from $P$. infestans can germinate on $S$. nigrum leaves but penetration is stopped by strong $\mathrm{HR}^{37,42}$. Rpi genes from plant species that only rarely support pathogen growth have likely not participated, or are no longer participating, in an evolutionary arms race with $P$. infestans, and hence, the pathogen's effectors have not (yet) evolved to evade detection by these Rpi genes. Under this scenario, a pre-existing standing variation in the pathogen for overcoming such Rpi genes is either absent or extremely rare. This makes such genes promising candidates for provision of broad-spectrum and durable late blight resistance, provided they are not deployed alone which facilitates one-step genetic changes in the pathogen to evade them, but rather in combination with other genes, as in the source plant ${ }^{43}$.

We report here a novel, broad-spectrum $S$. americanum resistance gene, Rpi-amrl. We also identified eight additional Rpi-amrl alleles from different $S$. americanum accessions and relatives, including one Rpi-amrl allele that translocated to the long arm of chromosome 1. Homology-based cloning also revealed the presence of functional Rpi-amrl homologs in S. nigrum. All nine cloned Rpi-amrl alleles confer late blight resistance in transient assays in N. benthamiana, and both Rpi-amr1-2272 and Rpiamr1-2273 in potato cv Maris Piper background confer resistance to all 19 tested $P$. infestans isolates from different countries, many of which overcome other Rpi genes. Thus, Rpi-amrl is widely distributed in germplasm of S. americanum, its relatives and S. nigrum, and may contribute to the resistance of nearly all accessions to $P$. infestans.

Many plant $R$ genes and their corresponding $A v r$ genes evolved differential recognition specificities with extensive allelic series for both $R$ gene and $A v r$ genes. Examples include ATR 1 and RPP1 or ATR13 and RPP13 from Hyaloperonospora arabidopsidis and Arabidopsis ${ }^{15}, A v r 567$ and $L$ genes from the rust Melampsora lini and flax ${ }^{44}$, and 
multiple and diverse recognized effectors from barley powdery mildew and Mla from barley. Similarly, Avramrl and its homologs from several $P$. infestans races ${ }^{31}$ were found to be differentially recognized by alleles of the Rpi-amrl gene. Remarkably though, Rpi-amrl nucleotide diversity of the $R$ gene did not show any of the hallmarks of diversifying or balancing selection.

Rather than through mutation accumulation, the high allelic variation observed at Rpiamrl appears to have been generated partly by recombination between significantly diverged alleles and paralogs. The recombination events are likely to be rare relative to the mutation rate, given that the alleles carry many polymorphisms. This evolutionary scenario can explain the observed mosaic-like structure of high and low sequence similarities when the Rpi-amrl alleles were mapped against the contig based on two overlapping BAC clones. The deep coalescence of alleles that is implicit in this scenario can be generated by balancing selection, but we did not find evidence of such selection when analysing the nucleotide substitution patterns. Recombination between Rpi-amrl alleles could have eroded this signature of selection, as has been observed also in $R p 1$ resistance genes in grasses ${ }^{45}$ and in the vertebrate immune genes of the major histocompatibility complex (MHC) ${ }^{46,47}$. Nucleotide sequence diversity across the Rpiamrl alleles is correlated with only slight differences in Avramrl recognition specificity. Rpi-amrl alleles can even recognize multiple Avramrl paralogs from a single $P$. infestans strain, a scenario that might elevate durability of resistance. Since the $S$. americanum population recognizes multiple Avramrl alleles and paralogs, small mutational changes in Avramrl gene are unlikely to suffice to escape detection, which makes resistance-breaking less likely, thus promoting evolutionary durability of Rpiamrl. Remarkably, Avramrl (PITG_07569) was recently reported to regulate plant alternative splicing and promote the colonization of $P$. infestans ${ }^{48}$, indicating Avramr1 contributes an important function for the virulence of $P$. infestans. We hypothesise that this enhanced recognition capacity could be key to the evolution of "non-host" resistance, offering an escape for the plant from the coevolutionary arms race. Conceivably, stacking Rpi-amrl alleles in cis could extend the recognition specificities, which could potentially lead to even more durable late blight resistance.

Intriguingly, two Avramrl homologs from $P$. parasitica and $P$. cactorum are recognized by all Rpi-amrl homologs. Presumably, these genes have been under even 
less selection pressure to evade Rpi-amrl recognition. This result indicates that Rpiamrl has the potential to provide "non-host" type resistance in $S$. americanum against multiple oomycete pathogens like $P$. parasitica and $P$. cactorum, which can infect a wide range of hosts. As both the resistance and effector recognition of Rpi-amrl are $N R C 2$ or $N R C 3$ dependent, co-expression of $N R C 2$ or NRC3 with Rpi-amrl might enable it to confer resistance to other Phytophthora species outside the Solanaceae.

In summary, we cloned Rpi-amrl, a broad-spectrum Rpi gene that contributes to the strong late blight resistance of nearly all $S$. americanum accessions to late blight. The apparent redundancy across the Rpi-amrl gene family may serve an evolutionary function by broadening the scope for recognizing multiple Avramrl alleles and paralogs, and potentially reducing the probability of evolution of resistance-breaking strains. Stacking this type of $R p i$ gene with additional Rpi genes might help to turn host plants such as potato into non-hosts for late blight, enabling broad-spectrum and durable resistance. 


\section{Materials and Methods:}

\section{Development of mapping populations}

Fourteen $P$. infestans resistant diploid Solanum americanum and relatives were used in this study (Table 1). The F1 populations were generated by crossing with a susceptible Solanum americanum accession 954750186 (working name SP2271) as a female parent. Heterozygous F1 progeny was allowed to self-pollinate to generate F2 segregating populations, or further back-crossed to the susceptible parent and allowed to self-pollinate until resistance to $P$. infestans co-segregated as a monogenic trait.

\section{$P$. infestans infection assay}

$P$. infestans isolates were cultured on rye and sucrose agar (RSA) medium at $18^{\circ} \mathrm{C}$ for 10 days. Sporangia were washed off with cold water and incubated at $4{ }^{\circ} \mathrm{C}$ for $1-2 \mathrm{~h}$ to induce zoospore release. Detached leaves were inoculated on the abaxial side with $10 \mu$ droplets of zoospore suspension (50-100,000 per $\mathrm{ml}$ ). The inoculated leaves were incubated at $18^{\circ} \mathrm{C}$ in high humidity under $16 \mathrm{~h}$ day/8 h night photoperiod conditions. Disease scoring was done at 5-7 days after infection.

\section{DNA and RNA extraction}

RenSeq experiments (both short- and long-reads protocols) were conducted on gDNA freshly extracted from young leaves using the DNeasy Plant Mini Kit (Qiagen) according to the manufacturer's protocol. For the cDNA RenSeq experiment, RNA was extracted using TRIReagent (Sigma-Aldrich, MO, USA) and Direct-zol RNA MiniPrep Kit (Zymo Research, CA, USA), following manufacturer's recommendations.

\section{Mapping of Rpi-amr1}

To map the underlying resistance gene from the resistant parent 954750184 (working name SP2273), we generated an F2 segregating population which was phenotyped with $P$. infestans isolates EC1_3626 and 06_3928A. Selected resistant plants were self-pollinated and up to 100 
plants from F3 populations were screened for resistance and susceptibility with $P$. infestans isolates EC1_3626 and 06_3928A.gDNA from susceptible F2 and F3 plants (BS pool), as well as gDNA from the resistant $(\mathrm{R})$ and susceptible parent $(\mathrm{S})$ were subjected to RenSeq using Solanaceae bait library ${ }^{27}$ and sequenced with Illumina MiSeq 250 bp paired-end reads. Preprocessing, assembly, mapping and SNP calling was performed as described earlier ${ }^{27,30}$.

The same gDNA samples were used in a RAD-seq experiment using PstI digestion and Illumina HiSeq sequencing, which was outsourced to Floragenex Inc. (OR, USA). Bioinformatic analysis was also performed by Floragenex using Solanum lycopersicum genome as a reference ${ }^{49}$. SNP calling resulted in sixteen polymorphic sites with eleven of them locating at the top of chromosome 11 (Supplementary table 1). The remaining ones were randomly distributed on chromosomes 4 and 1 .

We additionally outsourced Whole Genome Shotgun sequencing (WGS) of R and S samples to BGI (BGI, Shenzhen, China) for $~ 30$ deep Illumina HiSeq sequencing with 100PE. Reads from the resistant parent were assembled as described in $^{30}$ and we used our previously published in silico trait mapping pipelines to perform SNP calling and detection of polymorphisms linked to disease resistance ${ }^{27,30}$. Contigs polymorphic between $\mathrm{R}$ and $\mathrm{S}$ parents were further aligned to the DM reference genome to identify their position.

Screening a set of markers derived from these three approaches on gDNA of 94 susceptible F2 and F3 plants identified 12 markers linked to resistance response that flank the $R$ locus between $7.5 \mathrm{cM}$ to one side and $4.3 \mathrm{cM}$ to the other side (WGS, Table S1). Four of these markers were found to co-segregate with the resistance, and two others located around $1 \mathrm{cM}$ on either side, CAPS marker RAD_3 to the distal side and the PCR marker WGS_1 to the proximal side (Figure 1). Both $1 \mathrm{cM}$ markers were subsequently used to genotype 1,793 F2 plants, and we identified 228 recombinants (118 homozygous susceptible to one side and heterozygous to the other, 110 homozygous resistant to one side and heterozygous to the other).

The 118 informative recombinants (homozygous susceptible / heterozygous) were further genotyped using eight linked markers (Figure 1b), and tested in detached leaf assays for their response to $P$. infestans isolates EC1_3626 and 06_3928A. This revealed that markers CLC_3 (WGS_3) and RAD1 are flanking with a single recombination event for each marker, and CLC_2 (WGS_2), 56766 and 46418 are co-segregating with the resistance locus (Figure 1b). 
Comparison of the linkage map (Figure 1) with the potato reference genome ${ }^{34}$ identified the homogeneous CNL-3 NLR gene sub-family to be within the co-segregating locus. This cluster comprises 18 members on potato reference chromosome 11 .

\section{BAC clones identification and analysis}

Construction and screening of 5x BAC library from resistant parent SP2273 was outsourced to BioS\&T company (Quebec, Canada). Two candidate BAC clones (5G and $12 \mathrm{H}$ ) were identified in PCR screen with WGS_2 marker-specific primers. BAC sequencing with RSII PacBio platform and bioinformatic analysis was outsourced to Earlham Institute (Norwich, UK); both BACs were assembled into single contigs with length of $117,865 \mathrm{bp}$ (5G) and $117,666 \mathrm{bp}(12 \mathrm{H})$. While the co-segregating marker WGS_2 was present on both BAC clones, a further co-segregating marker WGS_3 was only present on $12 \mathrm{H}$. The BACs were further assembled into one 204,128 bp contig (available in GenBank under study number MW348763). NLRs on the contig sequence were annotated using NLR-annotator ${ }^{50}$ and Geneious 8.1.2 build-in ORF prediction tool. Gene models were annotated manually using cDNA RenSeq data generated from S. americanum accession SP2273 as described below.

\section{3' RACE}

Total RNA was extracted using RNeasy Plant Mini Kit (Qiagen) and treated with RNase-Free DNase (Qiagen) following manufacturer's instructions. First strand cDNA was synthesized from total RNA using SuperScript ${ }^{\mathrm{TM}}$ First-Strand Synthesis System for RT-PCR (Invitrogen, CA, USA) with P7-oligoDT primer. The resulting product was amplified with P7- and gene specific primers by using KAPA HiFi HotStart ReadyMix PCR Kit (Kapa Biosystems, Cape Town, SA) and cloned into $\mathrm{pCR}^{\mathrm{TM}}$-Blunt II-TOPO vector by using Zero Blunt ${ }^{\circledR} \mathrm{TOPO}^{\circledR}$ PCR Cloning Kit (Invitrogen) and transformation was performed using One Shot ${ }^{\mathrm{TM}}$ TOP10 Chemically Competent E. coli (Invitrogen). Isolation of plasmid DNA was performed with NucleoSpin ${ }^{\circledR}$ Plasmid kit (MACHEREY-NAGEL, Duren, Germany).

\section{Resistance gene enrichment sequencing (RenSeq) and Gene enrichment sequencing (GenSeq)}


SMRT RenSeq, short-read RenSeq and cDNA RenSeq were performed as described previously ${ }^{2}$ and enriched libraries were sequenced at Earlham Institute, Norwich, UK.

Illumina GenSeq was performed as described above (Illumina RenSeq), except GenSeq baits ${ }^{51}$ were used instead of RenSeq baits.

PacBio reads were processed and assembled using Geneious R8.1.8 ${ }^{52}$ as described $^{30}$. NLR coding sequences were predicted with Geneious and AUGUSTUS ${ }^{53}$ and annotated with NLR$\operatorname{parser}^{50}$.

To infer linked polymorphisms, the quality control for Illumina paired-end reads was performed using Trimmomatic ${ }^{54}$ with standard settings. For the RenSeq, the paired reads were mapped to PacBio-assembled contigs from the resistant parent, while GenSeq reads were mapped to the reference DM genome (PGSC_DM_v4.03_pseudomolecules.fasta), using BWA mapper $^{55}$ with default settings. PCR duplicates and unmapped reads were removed and Mpileup files to find out potential linked SNPs were created using SAMtools ${ }^{56}$. Mpileup files were processed with $\operatorname{VarScan}^{57}$ set to minimum read depth 20, minimum variant allele frequency threshold 0.1 , and minimum frequency to call homozygote 0.98 . The candidate SNPs were manually inspected using Savant genome browser ${ }^{58}$. TopHat ${ }^{59}$ with default settings was used to map cDNA Illumina reads to assembled PacBio data. All the tools used in this study were embedded in The Sainsbury Laboratory (TSL) customized Galaxy instance, if not stated otherwise.

\section{Transient complementation of a candidate genes in $N$. benthamiana}

The candidate genes were PCR amplified from gDNA with their own promoters (1-2 kb upstream of start codon) and up to $1 \mathrm{~kb}$ terminator elements, and cloned into USER vector as described $^{2}$. Transient complementation assays followed by P. infestans inoculation were performed as described ${ }^{2}$.

\section{Stable transformation of susceptible potato cultivar Maris Piper}

Stable transgenic plants with constructs carrying Rpi-amrl-2272, Rpi-amrl-2273 or Rpiamrla under the control of their native regulatory elements were created in the background of 
potato cultivar Maris Piper as described previously ${ }^{60}$. At least 10 independent transgenic lines were generated for each construct and tested for the presence of the transgene using gene specific primers. All positive Rpi-amrl-2272 and Rpi-amrl-2273 lines showed resistance in DLA with $P$. infestans isolate 88069 , while Rpi-amrla transgenic plants were fully susceptible. Selected lines of Rpi-amr1-2272 and Rpi-amrl-2273 were tested in DLA with 19 additional $P$. infestans isolates (Table 2). WT Maris Piper plants were used as a negative control.

\section{Generation of $N R C 2 / 3$ knockout $N$. benthamiana}

NRC4 and NRC2/NRC3/NRC4 knockout $N$. benthamiana lines were described previously ${ }^{35,36}$. Knocking out of NRC2/NRC3 in N. benthamiana were performed according to the methods described previously ${ }^{35}$. Forward primers CHW_sgNbNRCs and reverse primer JC_sgrna_R ${ }^{35}$ were used to clone sgRNA2.1-4, sgRNA3.1-4 into Golden Gate level 1 vectors for different positions. Constructs of sgRNAs targeting $N$. benthamiana NRC2 and NRC3 were assembled into level 2 vector pICSL4723 together with pICSL11017 (pICH47732::NOSp::BAR, Addgene no. 51145) and pICH47742::35S::Cas961. Leaf discs of $N$. benthamiana were transformed with the binary vector pICSL4723 containing the BAR selection marker gene, Cas9 expression cassette, and sgRNAs targeting NRC2 and NRC3. T0 transgenic plants were selected in the medium with phosphinothricin $(2 \mathrm{mg} / \mathrm{L})$ and then transferred into the soil. The progeny of the transformants were genotyped using amplicon sequencing as described previously $^{35}$ (Fig. S6a). T3 populations from the selected T2 plants were used for further experiments. $N R C 2 / 3$ knockout line (nrc23_1.3.1) did not exhibit any growth defects when compared to the wild type plants (Fig. S6b).

\section{Cloning Rpi-amr1 homologs from resistant Solanum nigrum accessions}

To test if Rpi-amrl also contributes to late blight resistance in S. nigrum, we firstly amplified and sequenced the first exon of Rpi-amrl from two resistant and one reported susceptible $S$. nigrum accessions. From two resistant accessions (SP1088 and SP1097; Table S4), we amplified sequences with $>99 \%$ nucleotide identity to S. americanum Rpi-amr1-2273.

To clone the full CDS of these Rpi-amrl homologs, we designed nested PCR primers based on Rpi-amr1-2273 sequence (First PCR: Rpi-amr1-77bp_F: 5' ATGTGGACATAAATCATTTAGACAA 3' and Rpi-amr1-USER_R: 5' GGTTTAAUTTACACAGTGCCGTCCTGCTT 3' ; Second PCR: Rpi-amr1-UF: 5' 
GGCTTAAUATGGCATATGCTGCTCTTTCTT 3' and Rpi-amr1-USER_R: 5' GGTTTAAUTTACACAGTGCCGTCCTGCTT 3'). The amplicons were cloned into USER vector with $35 \mathrm{~S}$ promoter as described ${ }^{2}$, the clones were sequenced and transformed into Agrobacterium strain AGL1 (pVirG), the transient HR and disease assays are as described previously.

\section{Phylogenetic tree construction}

Phylogenetic tree was generated from protein sequences of the cloned Solanaceae $R$ genes obtained from NCBI. Full-length sequences were aligned using ClustalW $1.74^{62}$ and the alignments were imported to the MEGA $7^{63}$ to build a maximum-likelihood phylogenetic tree with Jones-Taylor-Thornton (JTT) substitution model and 100 bootstraps.

\section{Evolutionary analyses of Rpi-amr1 and Avramr1 homologs}

CDS were aligned using MUSCLE ${ }^{64}$ as implemented in seaview ${ }^{65}$ with and without outgroup (the closest homologs from S. lycopersicum and P. cactorum for Rpi-amrl and Avramrl, respectively). Calculations of diversity statistics and the MacDonald-Kreitmann Test were executed through DNAsp5.066; DAMBE ${ }^{67}$ was used to rule out saturation. For Rpi-amrl homologs, the calculations were preformed separately on annotated full-length sequences as well as the individual domains.

We used $3 \mathrm{SEQ}^{68}$ to identify break points in the aligned CDS. To confirm gene conversion events in Rpi-amrl, we mapped the CDS back to the BAC_5G sequence using BLAT (minScore 1500, minMatch 93) ${ }^{69}$. The resulting .psl files were converted into .bed files using a custom R script, prior to visualization using the R package Sushi ${ }^{70}$.

\section{HybridCheck}

For each accession, FASTA files of all Rpi-amrle orthologs or Rpi-amrl paralogs in combinations of three (triplets) were generated and aligned using MUSCLE v3.8.31 ${ }^{64}$. The sequence triplets were analysed using HybridCheck ${ }^{40}$ to detect and date recombination blocks between $R$ pi-amrl orthologs (sliding windows $=200 \mathrm{bp}$ ) or paralogs (sliding windows $=100$ 
bp); non-informative sites were removed from the sequence triplets. Figures showing sequence similarity were plotted (MosaicScale $=50$ ) with HybridCheck and formatted using R v3.2.0 (https://www.r-project.org). The colour of each sequence window was calculated based on the proportion of SNPs shared between pairwise sequences at informative sites.

\section{Data availability}

Supporting raw reads were deposited in European Nucleotide Archive (ENA) under project number PRJEB38240. BAC and Rpi-amr1 allele sequences were deposited in GenBank under accession numbers MW345286-95 and MW348763. Detailed accession information are shown in Table S7. All the materials in this study are available upon request.

\section{References}

1. World Food and Agriculture - Statistical Pocketbook 2019. 1-254 (2019).

2. Saville, A. et al. Fungicide sensitivity of U.S. genotypes of Phytophthora infestans to six oomycete-targeted compounds. Plant Disease 99, 659-666 (2015).

3. Malcolmson, J. F. \& Black, W. New $R$ genes in Solanum demissum lindl. And their complementary races of Phytophthora infestans (Mont.) de bary. Euphytica 15, 199-203 (1966).

4. Park, T.-H. et al. The late blight resistance locus Rpi-bib3 from Solanum bulbocastanum belongs to a major late blight $R$ gene cluster on chromosome 4 of potato. MPMI 18, 722-729 (2005).

5. Huang, S. et al. Comparative genomics enabled the isolation of the $R 3 a$ late blight resistance gene in potato. The Plant Journal 42, 251-261 (2005).

6. Vossen, J. H. et al. The Solanum demissum $R 8$ late blight resistance gene is an $\mathrm{Sw}$ 5 homologue that has been deployed worldwide in late blight resistant varieties. Theor Appl Genet 129, 1785-1796 (2016).

7. Song, J. et al. Gene $R B$ cloned from Solanum bulbocastanum confers broad spectrum resistance to potato late blight. Proceedings of the National Academy of Sciences 100, 9128-9133 (2003).

8. van der Vossen, E. A. G. et al. The Rpi-blb2 gene from Solanum bulbocastanum is an Mi-1 gene homolog conferring broad-spectrum late blight resistance in potato. The Plant Journal 44, 208-222 (2005).

9. Pel, M. A. et al. Mapping and cloning of late blight resistance genes from Solanum venturii using an interspecific candidate gene approach. MPMI 22, 601-615 (2009).

10. Foster, S. J. et al. Rpi-vnt1.1, a Tm-22 homolog from Solanum venturii, confers resistance to potato late blight. MPMI 22, 589-600 (2009).

11. Jones, J. D. G., Vance, R. E. \& Dangl, J. L. Intracellular innate immune surveillance devices in plants and animals. Science 354, aaf6395 (2016).

12. $\mathrm{Wu}, \mathrm{C} .-\mathrm{H}$. et al. NLR network mediates immunity to diverse plant pathogens. Proceedings of the National Academy of Sciences 114, 8113-8118 (2017). 
13. Fry, W. Phytophthora infestans: the plant (and $R$ gene) destroyer. Molecular Plant Pathology 9, 385-402 (2008).

14. Jones, J. D. G. \& Dangl, J. L. The plant immune system. Nature 444, 323-329 (2006).

15. Rehmany, A. P. et al. Differential recognition of highly divergent downy mildew avirulence gene alleles by $R P P 1$ resistance genes from two Arabidopsis lines. The Plant Cell 17, 1839-1850 (2005).

16. Vleeshouwers, V. G. A. A. et al. Effector genomics accelerates discovery and functional profiling of potato disease resistance and Phytophthora infestans avirulence genes. PLoS ONE 3, e2875 (2008).

17. Haas, B. J. et al. Genome sequence and analysis of the Irish potato famine pathogen Phytophthora infestans. Nature 461, 393-398 (2009).

18. Armstrong, M. R. et al. An ancestral oomycete locus contains late blight avirulence gene $A v r 3 a$, encoding a protein that is recognized in the host cytoplasm. Proceedings of the National Academy of Sciences 102, 7766-7771 (2005).

19. Stam, R., Silva Arias, G. A. \& Tellier, A. Subsets of NLR genes show differential signatures of adaptation during colonization of new habitats. New Phytol 224, 367-379 (2019).

20. Van de Weyer, A.-L. et al. A species-wide inventory of NLR genes and alleles in Arabidopsis thaliana. Cell 178, 1260-1272.e14 (2019).

21. McDowell, J. M. et al. Intragenic recombination and diversifying selection contribute to the evolution of downy mildew resistance at the RPP8 locus of Arabidopsis. The Plant Cell 10, 1861-1874 (1998).

22. Liu, J. et al. Genetic variation and evolution of the Pi9 blast resistance locus in the AA genome Oryza species. J. Plant Biol. 54, 294-302 (2011).

23. Ellis, J. G., Lawrence, G. J., Luck, J. E. \& Dodds, P. N. Identification of regions in alleles of the flax rust resistance gene $L$ That determine differences in gene-forgene specificity. The Plant Cell 11, 495-506 (1999).

24. Seeholzer, S. et al. Diversity at the Mla powdery mildew resistance locus from cultivated barley reveals sites of positive selection. MPMI 23, 497-509 (2010).

25. Saur, I. M. et al. Multiple pairs of allelic MLA immune receptor-powdery mildew AVRA effectors argue for a direct recognition mechanism. eLife 8, 1957 (2019).

26. Anderson, C. et al. Genome analysis and avirulence gene cloning using a highdensity RADseq linkage map of the flax rust fungus, Melampsora lini. BMC Genomics 17, 1-20 (2016).

27. Jupe, F. et al. Resistance gene enrichment sequencing (RenSeq) enables reannotation of the $N B-L R R$ gene family from sequenced plant genomes and rapid mapping of resistance loci in segregating populations. The Plant Journal 76, 530 544 (2013).

28. Thilliez, G. J. A. et al. Pathogen enrichment sequencing (PenSeq) enables population genomic studies in oomycetes. New Phytol 4, 903 (2018).

29. Jouet, A. et al. Albugo candida race diversity, ploidy and host-associated microbes revealed using DNA sequence capture on diseased plants in the field. New Phytol 93, 959 (2018).

30. Witek, K. et al. Accelerated cloning of a potato late blight-resistance gene using RenSeq and SMRT sequencing. Nature Biotechnology 34, 656-660 (2016).

31. Lin, X. et al. Identification of Avramr1 from Phytophthora infestans using long read and cDNA pathogen-enrichment sequencing (PenSeq) Molecular Plant Pathology doi: https://doi.org/10.1111/mpp.12987. 
32. Kamoun, S., van West, P., Vleeshouwers, V. G. A. A., de Groot, K. E. \& Govers, F. Resistance of Nicotiana benthamiana to Phytophthora infestans is mediated by the recognition of the elicitor protein INF1. The Plant Cell 10, 1413-1425 (1998).

33. Grund, E., Tremousaygue, D. \& Deslandes, L. Plant NLRs with integrated domains: unity makes strength. Plant Physiol 179, 1227-1235 (2019).

34. Xu, X. et al. Genome sequence and analysis of the tuber crop potato. Nature 475, 189-195 (2011).

35. Wu, C.-H. et al. NRC4 Gene cluster Is not essential for bacterial flagellin-triggered immunity. Plant Physiol. 182, 455-459 (2020).

36. Adachi, H. et al. An N-terminal motif in NLR immune receptors is functionally conserved across distantly related plant species. eLife 8, 121 (2019).

37. Colon, L. T. et al. Resistance to potato late blight (Phytophthora infestans (Mont.) de Bary) in Solanum nigrum, S. villosum and their sexual hybrids with $S$. tuberosum and S. demissum. Euphytica 66, 55-64 (1992).

38. Lebecka, R. Host-pathogen interaction between Phytophthora infestans and Solanum nigrum, S. villosum, and S. scabrum. Eur J Plant Pathol 120, 233-240 (2007).

39. Poczai, P. \& Hyvönen, J. On the origin of Solanum nigrum: can networks help? Mol Biol Rep 38, 1171-1185 (2010).

40. Ward, B. J. \& van Oosterhout, C. HYBRIDCHECK: software for the rapid detection, visualization and dating of recombinant regions in genome sequence data. Molecular Ecology Resources 16, 534-539 (2016).

41. Vleeshouwers, V. G. A. A. et al. Understanding and exploiting late blight resistance in the age of effectors. Annu. Rev. Phytopathol. 49, 507-531 (2011).

42. Vleeshouwers, V. G. A. A., van Dooijeweert, W., Govers, F., Kamoun, S. \& Colon, L. T. The hypersensitive response is associated with host and nonhost resistance to Phytophthora infestans. Planta 210, 853-864 (2000).

43. Jones, J. D. G. et al. Elevating crop disease resistance with cloned genes. Philos. Trans. R. Soc. Lond., B, Biol. Sci. 369, 20130087-20130087 (2014).

44. Dodds, P. N. et al. Direct protein interaction underlies gene-for-gene specificity and coevolution of the flax resistance genes and flax rust avirulence genes. Proceedings of the National Academy of Sciences 103, 8888-8893 (2006).

45. Jouet, A., McMullan, M. \& van Oosterhout, C. The effects of recombination, mutation and selection on the evolution of the Rpl resistance genes in grasses. Mol. Ecol. 24, 3077-3092 (2015).

46. Ohta, T. Gene conversion vs point mutation in generating variability at the antigen recognition site of major histocompatibility complex loci. Journal of Molecular Evolution 41, 115-119 (1995).

47. Spurgin, L. G. et al. Gene conversion rapidly generates major histocompatibility complex diversity in recently founded bird populations. Mol. Ecol. 20, 5213-5225 (2011).

48. Huang, J. et al. Phytophthora effectors modulate genome-wide alternative splicing of host mRNAs to reprogram plant immunity. Molecular Plant 1-46 (2020). doi:10.1016/j.molp.2020.07.007

49. Sato, S. et al. The tomato genome sequence provides insights into fleshy fruit evolution. Nature 485, 635-641 (2012).

50. Steuernagel, B., Jupe, F., Witek, K., Jones, J.2015. NLR-parser: rapid annotation of plant NLR complements. Bioinformatics 1665-1667 (2015). 
51. Chen, X. et al. Identification and rapid mapping of a gene conferring broadspectrum late blight resistance in the diploid potato species Solanum verrucosum through DNA capture technologies. Theor Appl Genet 131, 1287-1297 (2018).

52. Kearse, M. et al. Geneious Basic: An integrated and extendable desktop software platform for the organization and analysis of sequence data. Bioinformatics 28, 1647-1649 (2012).

53. Stanke, M. \& Morgenstern, B. AUGUSTUS: a web server for gene prediction in eukaryotes that allows user-defined constraints. Nucleic Acids Research 33, W465-W467 (2005).

54. Bolger, A. M., Lohse, M. \& Usadel, B. Trimmomatic: a flexible trimmer for Illumina sequence data. Bioinformatics 30, 2114-2120 (2014).

55. Li, H. \& Durbin, R. Fast and accurate short read alignment with Burrows-Wheeler transform. Bioinformatics 25, 1754-1760 (2009).

56. Li, H. et al. The Sequence Alignment/Map format and SAMtools. Bioinformatics 25, 2078-2079 (2009).

57. Koboldt, D. C. et al. VarScan 2: Somatic mutation and copy number alteration discovery in cancer by exome sequencing. Genome Research 22, 568-576 (2012).

58. Fiume, M., Williams, V., Brook, A. \& Bioinformatics, M. B. Savant: genome browser for high-throughput sequencing data. Bioinformatics 25, 1938-1944 (2010).

59. Trapnell, C., Pachter, L. \& Salzberg, S. L. TopHat: discovering splice junctions with RNA-Seq. Bioinformatics 25, 1105-1111 (2009).

60. Kumar, A., Taylor, M. A., Arif, S. A. M. \& Davies, H. V. Potato plants expressing antisense and sense S-adenosylmethionine decarboxylase (SAMDC) transgenes show altered levels of polyamines and ethylene: antisense plants display abnormal phenotypes. The Plant Journal 9, 147-158 (1996).

61. Castel, B. et al. Diverse NLR immune receptors activate defence via the RPW8NLR NRG1. New Phytol 222, 966-980 (2019).

62. Thompson, J. D., Higgins, D. G. \& Gibson, T. J. CLUSTAL W: improving the sensitivity of progressive multiple sequence alignment through sequence weighting, position-specific gap penalties and weight matrix choice. Nucleic Acids Research 22, 4673-4680 (1994).

63. Kumar, S., Nei, M., Dudley, J. \& Tamura, K. MEGA: A biologist-centric software for evolutionary analysis of DNA and protein sequences. Briefings in Bioinformatics 9, 299-306 (2008).

64. Edgar, R. C. MUSCLE: multiple sequence alignment with high accuracy and high throughput. Nucleic Acids Research 32, 1792-1797 (2004).

65. Gouy, M., Guindon, S., and, O. G. M. B.2010. SeaView version 4: a multiplatform graphical user interface for sequence alignment and phylogenetic tree building. Mol Biol Evol (2009).

66. Rozas, J. \& Sánchez-DelBarrio, J. C. DnaSP, DNA polymorphism analyses by the coalescent and other methods. Bioinformatics (2009).

67. Xia, X. DAMBE: software package for data analysis in molecular biology and evolution. Mol Biol Evol (2013).

68. Ratmann, O., Lam, H. M. \& Boni, M. F. Improved algorithmic complexity for the 3SEQ recombination detection algorithm. Mol Biol Evol (2017).

69. Kent, W. J. BLAT--the BLAST-like alignment tool. Genome Research 12, 656664 (2002).

70. Phanstiel DH (2020). Sushi: Tools for visualizing genomics data. R (1.26.0.) 
71. Champouret, N. et al. Phytophthora infestans isolates lacking class I ipiO variants are virulent on Rpi-blbl potato. MPMI 22, 1535-1545 (2009).

72. Cooke, D. E. L. et al. Genome analyses of an aggressive and invasive lineage of the Irish potato famine pathogen. PLoS Pathog 8, e1002940 (2012).

\section{Acknowledgements}

This research was financed from BBSRC grant BB/P021646/1 and the Gatsby Charitable Foundation. This research was supported in part by the NBI Computing infrastructure for Science (CiS) group through the provision of a High-Performance Computing Cluster. We would like to thank TSL bioinformatics team, transformation team and horticultural team for their support. We thank Experimental Garden and Genebank of Radboud University, Nijmegen, The Netherlands, IPK Gatersleben, Germany and Sandra Knapp (Natural History Museum, London, UK) for access to $S$. americanum, S. nigrescens and S. nigrum genetic diversity, and Geert Kessel, Francine Govers and Paul Birch for providing P. infestans isolates.

\section{Author contributions:}

K.W., X.L., F.J., R.S., C.O. and J.D.G.J. designed the study. K.W., X.L., H.S.K., F.J., A.I.W., S.B., R.H., W.B., L.T. and T.S., performed the experiments. K.W., X.L., H.S.K., F.J., A.I.W., B.S., R.S., C.O., S.F., and J.M.C. analysed the data. K.W., X.L., H.S.K., F.J. and J.D.G.J. wrote the manuscript with input from all authors. V.G.A.A.V., B.B.H.W, C.-H.W., H.A. and S.K. contributed resources. K.W., X.L and H.S.K. made equivalent contributions and should be considered joint first authors. All authors approved the manuscript.

\section{Conflict of interest:}

K.W., H.S.K., F.G.J. and J.D.G.J. are named inventors on a patent application (PCT/US2017/066691) pertaining to Rpi-amrl that was filed by the 2Blades Foundation on behalf of the Sainsbury Laboratory. The other authors declare no competing interests.

\section{Figure Legends:}

Fig. 1. Map-based cloning of Rpi-amrl and its resistance to $P$. infestans. 
(a) Mapping of Rpi-amrl in a small F2 population ( $\mathrm{n}=188$ gametes); the names of the markers and genetic distances are shown above or below the bar.

(b) Fine mapping of Rpi-amrl in the F2 population of 3,586 gametes. The names of the markers and the number of recombinants are shown above or below the bar.

(c) Physical map of the target Rpi-amrl interval based on the assembled BAC contig. The markers present on the BAC are shown. The predicted NLR genes are depicted as black arrows (expressed NLRs) or empty arrows (pseudogenized NLRs). Rpi-amrl (formerly Rpi-amrle) is indicated by a red arrow.

(d) Four Rpi-amrl transcripts detected by 3' RACE PCR.

(e) Leaves of $N$. benthamiana plants were infiltrated with the binary vector pICSLUS0003::35S overexpressing either the late blight resistance gene Rpiamr3 (positive control), one of seven Rpi-amrl candidates, or the non-functional Rpiamr3-S (negative control). Leaves were inoculated with $P$. infestans strain $8806924 \mathrm{~h}$ after infiltration. Only leaves infiltrated with Rpi-amr3 and Rpi-amrle (pictured) showed reduced pathogen growth, whereas $P$. infestans grew well in the presence of the remaining Rpi-amrl candidates. Only Rpi-amrlc is shown as the phenotype of all other non-functional candidate genes was indistinguishable. Photographs were taken 9 dpi.

(f) Transgenic potato cv. Maris Piper which expresses Rpi-amrl under the native regulatory elements is resistant to $P$. infestans isolate 88069 (top), displaying no symptoms at the spot of inoculation. Each leaflet was inoculated with a droplet containing approximately 1,000 zoospores; photographs were taken 9 dpi.

(g) The control plants carrying the non-functional candidate Rpi-amrla show large necrotic lesions and sporulation. Each leaflet was inoculated with a droplet containing approximately 1,000 zoospores; photographs were taken 9 dpi.

Fig. 2. Schematic representation of amino acid sequence alignment of Rpi-amr1 homologs (a) and $P$. infestans resistance in transient assay (b).

(a) The exons and the conserved NLR domains are highlighted at the top of the alignment (exons, grey; CC, orange; NB-ARC, blue; LRR, green). Black bars in the alleles indicate the polymorphic amino acids and indels as compared with Rpi-amrl-2273. The 
numbers next to the alleles refer to the accession working numbers (Table 1). Figure drawn to the scale.

(b) Nine Rpi-amrl homologs provide resistance to $P$. infestans in transient complementation assay. Rpi-amrl genes with native regulatory elements were infiltrated into $N$. benthamiana leaves. At 1 dpi, leaves were cut off and drop inoculated with $10 \mu$ of zoospore suspension $(50,000$ zoospores $/ \mathrm{mL})$ from $P$. infestans isolate 88069. The non-functional Rpi-amrl-2271 homolog from susceptible accession SP2271 was used as negative control. Photographs were taken 8 dpi.

\section{Fig. 3. Differential recognition of Rpi-amr1 and Avramr1 homologs.}

Four Avramrl homologs representing clades A-D, and $P$. parasitica and $P$. cactorum homologs were co-infiltrated with ten Rpi-amrl homologs, including a non-functional homolog Rpiamr1-2271, into $N$. benthamiana leaves. Colours from green to brown represent the strength of HR scored from 0 to 2 (see bottom panel). $\mathrm{N}=3$. The representative HR phenotype and scoring are shown in Fig. S3.

Left: phylogenetic tree of nine functional Rpi-amrl homologs and non-functional homolog Rpi-amr1-2271. Top: phylogenetic tree of Avramrl homologs from four isolates of P. infestans. * Stable Rpi-amrl-2307 N. benthamiana transformants show HR upon transient expression of Avramrl and Avramr1-13B1.

\section{Fig. 4. Rpi-amr1 is NRC2 or NRC3 dependent.}

(a) Phylogenetic analysis of Rpi-amr1 protein and other functional Solanaceae NLR proteins. The NLR clades shown here are as described previously ${ }^{30}$, the NRCdependent sensor clades are marked by blue box.

(b) Transient expression of Rpi-amrl-2273 in NRC2/NRC3 double knockout $N$. benthamiana, followed by zoospore inoculation of $P$. infestans isolate 88069 , results in large necrotic lesions indicating lack of resistance.

(c) Transient expression of Rpi-amr1-2273 in NRC2/NRC3/NRC4 triple knockout $N$. benthamiana, followed by zoospore inoculation of $P$. infestans isolate 88069 , results in large necrotic lesions indicating lack of the resistance.

(d) Transient expression of Rpi-amr1-2273 in NRC4 knockout $N$. benthamiana, followed by zoospore inoculation of $P$. infestans isolate 88069 results in small necrotic lesions indicating resistance. 


\section{Tables:}

Table 1. S. americanum, S. nodiflorum and S. nigrescens accessions used in this study and the corresponding Rpi-amr1 homologs

Table 1

\begin{tabular}{|c|c|c|c|c|c|c|c|c|}
\hline Accession & $\begin{array}{l}\text { Workin } \\
\text { g name }\end{array}$ & Species & $\begin{array}{l}\text { Repo } \\
\text { rted } \\
\text { origi } \\
\text { n }\end{array}$ & $\begin{array}{l}\text { Sour } \\
\text { ce }\end{array}$ & $\begin{array}{l}\text { Late } \\
\text { blight } \\
\text { resistance }\end{array}$ & $\begin{array}{l}\text { Rpi-amr1 } \\
\text { homolog }\end{array}$ & $\begin{array}{l}\text { Simila } \\
\text { rity }\end{array}$ & Cloning method \\
\hline 954750186 & SP2271 & S. americanum & Brazil & RU & $\begin{array}{l}\text { Susceptibl } \\
\text { e }\end{array}$ & & & \\
\hline 954750184 & SP2273 & $\begin{array}{l}\text { S. americanum } \\
\text { var. patulum }\end{array}$ & $\begin{array}{l}\text { unkno } \\
\text { wn }\end{array}$ & $\mathrm{RU}$ & Resistant & $\begin{array}{l}\text { Rpi-amrl- } \\
2273\end{array}$ & $100 \%$ & $\begin{array}{l}\text { Map-based } \\
\text { cloning }\end{array}$ \\
\hline $\operatorname{sn} 27$ & SP1032 & $\begin{array}{l}\text { S. americanum } \\
\text { sensu lato }\end{array}$ & China & BGS & Resistant & $\begin{array}{l}\text { Rpi-amrl- } \\
1032\end{array}$ & $92.8 \%$ & $\begin{array}{l}\text { Association } \\
\text { genomics }\end{array}$ \\
\hline Veg422 & SP1034 & $\begin{array}{l}\text { S. americanum } \\
\text { sensu lato }\end{array}$ & $\begin{array}{l}\text { unkno } \\
\text { wn }\end{array}$ & $\mathrm{NN}$ & Resistant & $\begin{array}{l}\text { Rpi-arm1- } \\
2273\end{array}$ & $100 \%$ & $\begin{array}{l}\text { Association } \\
\text { genomics }\end{array}$ \\
\hline A54750014 & SP1101 & $\begin{array}{l}\text { S. americanum } \\
\text { sensu lato }\end{array}$ & $\begin{array}{l}\text { unkno } \\
\text { wn }\end{array}$ & RU & Resistant & $\begin{array}{l}\text { Rpi-amrl- } \\
1101\end{array}$ & $89.4 \%$ & SMRT RenSeq \\
\hline A14750006 & SP1123 & $\begin{array}{l}\text { S. americanum } \\
\text { sensu lato }\end{array}$ & $\begin{array}{l}\text { unkno } \\
\text { wn }\end{array}$ & RU & Resistant & $\begin{array}{l}\text { Rpi-amrl- } \\
1123\end{array}$ & $91.8 \%$ & $\begin{array}{l}\text { Association } \\
\text { genomics }\end{array}$ \\
\hline 954750174 & SP2272 & S. americanum & $\begin{array}{l}\text { unkno } \\
\text { wn }\end{array}$ & RU & Resistant & $\begin{array}{l}\text { Rpi-amrl- } \\
2272\end{array}$ & $89.4 \%$ & $\begin{array}{l}\text { Association } \\
\text { genomics }\end{array}$ \\
\hline SOLA 226 & SP2300 & S. americanum & Cuba & IPK & Resistant & $\begin{array}{l}\text { Rpi-amrl- } \\
2300\end{array}$ & $90.4 \%$ & SMRT RenSeq \\
\hline SOLA 425 & SP2307 & S. americanum & $\begin{array}{l}\text { Amer } \\
\text { ica }\end{array}$ & IPK & Resistant & $\begin{array}{l}\text { Rpi-amrl- } \\
2307\end{array}$ & $91.7 \%$ & $\begin{array}{l}\text { Association } \\
\text { genomics }\end{array}$ \\
\hline Wang 2059 & SP2360 & S. americanum & China & $\begin{array}{l}\mathrm{NH} \\
\mathrm{M}\end{array}$ & Resistant & $\begin{array}{l}\text { Rpi-arm1- } \\
2273\end{array}$ & $100 \%$ & $\begin{array}{l}\text { Association } \\
\text { genomics }\end{array}$ \\
\hline A14750138 & SP3399 & S. americanum & $\begin{array}{l}\text { unkno } \\
\text { wn }\end{array}$ & $\mathrm{RU}$ & Resistant & $\begin{array}{l}\text { Rpi-amrl- } \\
2272\end{array}$ & $89.4 \%$ & $\begin{array}{l}\text { Association } \\
\text { genomics }\end{array}$ \\
\hline A14750130 & SP3400 & S. nodiflorum & $\begin{array}{l}\text { unkno } \\
\text { wn }\end{array}$ & RU & Resistant & $\begin{array}{l}\text { Rpi-amrl- } \\
2273\end{array}$ & $100 \%$ & $\begin{array}{l}\text { Association } \\
\text { genomics }\end{array}$ \\
\hline 944750261 & SP3406 & S. nigrescens & $\begin{array}{l}\text { Bolivi } \\
\text { a }\end{array}$ & RU & Resistant & $\begin{array}{l}\text { Rpi-amrl- } \\
3406\end{array}$ & $92.5 \%$ & $\begin{array}{l}\text { Association } \\
\text { genomics }\end{array}$ \\
\hline
\end{tabular}




$\begin{array}{lllllllll}954750172 & \text { SP3408 } & \text { S. nigrescens } & \begin{array}{l}\text { Bolivi } \\ \text { a }\end{array} & \text { RU } & \text { Resistant } & \begin{array}{l}\text { Rpi-amrl- } \\ 3408\end{array} & 92.6 \% & \begin{array}{l}\text { Association } \\ \text { genomics }\end{array} \\ \text { A14750423 } & \text { SP3409 } & \text { S. nigrescens } & \begin{array}{l}\text { Mauri } \\ \text { tius }\end{array} & \text { RU } & \text { Resistant } & \begin{array}{l}\text { Rpi-amrl- } \\ 3409\end{array} & \text { 89.5\% } & \text { SMRT RenSeq }\end{array}$

RU - Radboud University, Nijmegen, The Netherlands

IPK - IPK Gatersleben, Germany

NHM - Natural History Museum, London, United Kingdom

BGS - Shanghai Botanical Garden, Shanghai, China

NN - Nicky's Nursery Ltd, Kent, United Kingdom

Table 2. Phenotypes of potato plants stably transformed with Rpi-amr1-2272 and Rpiamr1-2273 after inoculation with multiple isolates of $P$. infestans.

\begin{tabular}{|c|c|c|c|c|c|}
\hline Isolate & $\begin{array}{l}\text { Rpi-amrl- } \\
2272\end{array}$ & $\begin{array}{l}\text { Rpi-amrl- } \\
2273\end{array}$ & $\begin{array}{l}\text { Maris } \\
\text { Piper }\end{array}$ & Origin & Race $^{\mathrm{e}}$ \\
\hline NL00228 & $\mathrm{R}$ & $\mathrm{R}$ & $\mathrm{S}$ & The Netherlands & 1.2 .4 .7 \\
\hline US23 & $\mathrm{R}$ & $\mathrm{R}$ & $\mathrm{S}$ & USA & n.a. \\
\hline $3928 A^{a}$ & $\mathrm{R}$ & $\mathrm{R}$ & $\mathrm{S}$ & UK & $1 \cdot 2 \cdot 3 \cdot 4 \cdot 5 \cdot 6 \cdot 7 \cdot 10 \cdot 11^{\mathrm{f}}$ \\
\hline $\mathrm{EC} 3626^{\mathrm{b}}$ & $\mathrm{R}$ & $\mathrm{R}$ & $S$ & Ecuador & n.a. \\
\hline NL14538 & $\mathrm{R}$ & $\mathrm{R}$ & $\mathrm{S}$ & The Netherlands & n.a. \\
\hline $\mathrm{NR}_{47 \mathrm{UH}^{\mathrm{d}}}$ & $\mathrm{R}$ & $\mathrm{R}$ & $\mathrm{S}$ & UK & $1.3 \cdot 4 \cdot 7 \cdot 10.11^{\mathrm{f}}$ \\
\hline T30-4 & $\mathrm{R}$ & $\mathrm{R}$ & $\mathrm{S}$ & The Netherlands & n.a. \\
\hline USA618 & $\mathrm{R}$ & $\mathrm{R}$ & $S$ & USA & 1.2.3.6.7.10.11 \\
\hline KPI15-10 & $\mathrm{R}$ & $\mathrm{R}$ & $S$ & Korea & n.a. \\
\hline IPO-C & $\mathrm{R}$ & $\mathrm{R}$ & $\mathrm{S}$ & Belgium & 1.2.3.4.5.6.7.10.11 \\
\hline PIC99189 & $\mathrm{R}$ & $\mathrm{R}$ & $S$ & Mexico & 1.2.5.7.10.11 \\
\hline UK7824 & $\mathrm{R}$ & $\mathrm{R}$ & $S$ & UK & n.a. \\
\hline PIC99177 & $\mathrm{R}$ & $\mathrm{R}$ & $S$ & Mexico & 1.2.3.4.7.9.11 \\
\hline VK98014 & $\mathrm{R}$ & $\mathrm{R}$ & $\mathrm{S}$ & The Netherlands & 1.2.4.11 \\
\hline NL08645 & $\mathrm{R}$ & $\mathrm{R}$ & S & The Netherlands & n.a. \\
\hline PIC99183 & $\mathrm{R}$ & $\mathrm{R}$ & $S$ & Mexico & 1.2.3.4.5.7.8.10.11 \\
\hline NL11179 & $\mathrm{R}$ & $\mathrm{R}$ & $\mathrm{S}$ & The Netherlands & n.a. \\
\hline $\mathrm{EC} 1^{\mathrm{b}}$ & $\mathrm{R}$ & $\mathrm{R}$ & $S$ & Ecuador & 1.3.4.7.10.11 \\
\hline NL01096 & $\mathrm{R}$ & $\mathrm{R}$ & $\mathrm{S}$ & The Netherlands & 1.3.4.7.8.10.11 \\
\hline \multicolumn{6}{|c|}{$\begin{array}{l}\text { a Clonal lineage EU_13_A2, or "Blue13" } \\
\text { b Overcomes Rpi-vnt1 } \\
\text { c Overcomes Rpi-vnt1 and partially Rpi-blb1, Rpi-blb2 } \\
\text { d Clonal lineage EU_6_A1, commonly known as "Pink6" } \\
\text { e Summarized in }\end{array}$} \\
\hline
\end{tabular}


${ }^{\mathrm{f}} \mathrm{See}^{72}$ 